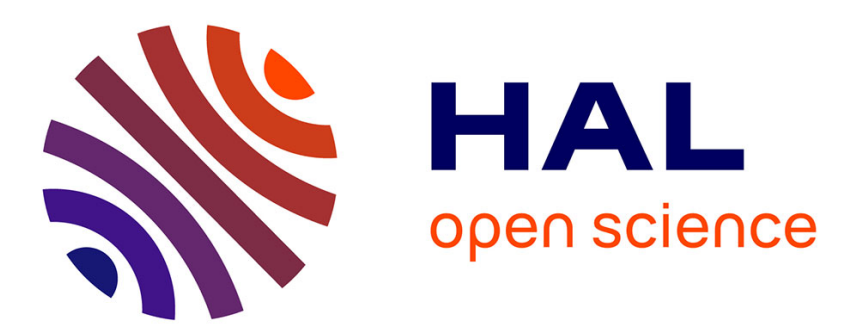

\title{
A fully predictive model for one-dimensional light attenuation by Chlamydomonas reinhardtii in a torus photobioreactor
}

L. Pottier, Jeremy Pruvost, J. Deremetz, Jack Legrand, C.G. G Dussap, J.F. Cornet

\section{To cite this version:}

L. Pottier, Jeremy Pruvost, J. Deremetz, Jack Legrand, C.G. G Dussap, et al.. A fully predictive model for one-dimensional light attenuation by Chlamydomonas reinhardtii in a torus photobioreactor. Biotechnology and Bioengineering, 2005, 91 (5), pp.569-582. 10.1002/bit.20475 . hal-02534059

\section{HAL Id: hal-02534059 \\ https://hal.science/hal-02534059}

Submitted on 14 Apr 2020

HAL is a multi-disciplinary open access archive for the deposit and dissemination of scientific research documents, whether they are published or not. The documents may come from teaching and research institutions in France or abroad, or from public or private research centers.
L'archive ouverte pluridisciplinaire HAL, est destinée au dépôt et à la diffusion de documents scientifiques de niveau recherche, publiés ou non, émanant des établissements d'enseignement et de recherche français ou étrangers, des laboratoires publics ou privés. 


\title{
A Fully Predictive Model for \\ One-Dimensional Light Attenuation by Chlamydomonas reinhardtii in a Torus Photobioreactor
}

\author{
L. Pottier, ${ }^{1}$ J. Pruvost, ${ }^{1}$ J. Deremetz, ${ }^{2}$ J.-F. Cornet ${ }^{2}$ J. Legrand, ${ }^{1}$ C.G. Dussap ${ }^{2}$ \\ ${ }^{1}$ GEPEA Laboratory, UMR-CNRS 6144, University of Nantes, \\ Faculté des Sciences et des Techniques, 2 rue de la Houssinière, \\ BP 92208, 44322 NANTES Cedex 3, France; telephone: +33 (0)2 511255 09; \\ fax: +33 (0)2 511255 05; e-mail: jeremy.pruvost@univ-nantes.fr \\ ${ }^{2}$ Laboratoire de Génie Chimique et Biochimique, \\ Université Blaise Pascal-Bât. CUST. 24 avenue des Landais, \\ BP 206, F 63174 AUBIERE Cedex, France
}

\begin{abstract}
The light attenuation in a photobioreactor is determined using a fully predictive model. The optical properties were first calculated, using a data bank of the literature, from only the knowledge of pigments content, shape, and size distributions of cultivated cells which are a function of the physiology of the current species. The radiative properties of the biological turbid medium were then deduced using the exact Lorenz-Mie theory. This method is experimentally validated using a large-size integrating sphere photometer. The radiative properties are then used in a rectangular, one-dimensional two-flux model to predict radiant light attenuation in a photobioreactor, considering a quasi-collimated field of irradiance. Combination of this radiative model with the predictive determination of optical properties is finally validated by in situ measurement of attenuation profiles in a torus photobioreactor cultivating the microalgae Chlamydomonas reinhardtii, after a complete and proper characterization of the incident light flux provided by the experimental set-up. (c) 2005 Wiley Periodicals, Inc.

Keywords: light transfer; predictive model; photobioreactor; Chlamydomonas reinhardtii; two-flux method; optical radiative properties
\end{abstract}

\section{INTRODUCTION}

Photosynthetic microorganisms like microalgae are known to be of interest in various areas including food, cosmetics, the environment, and even energy. For example, Chlamydomonas reinhardtii can produce hydrogen gas from water and (sun)light (Melis et al., 2000). However, large-scale cultivation in photobioreactors of photo-autotrophic microorganisms is still limited mainly because of the complex running of such kind of processes. As a consequence, the study and modeling of photobioreactors is a problem of increasing relevance. The main difficulty is to relate the light energy available in the culture to the growth by a predictive approach

Correspondence to: J. Pruvost that implies to first accurately describe the light transfer inside the photobioreactor volume. For this purpose, models of various complexities are described in the literature (Acien Fernandez et al., 1997; Cornet et al., 1998; Csogör et al., 2001; Suh and Lee, 2003; Tredici and Zitelli, 1998; Yun and Park, 2003). For an accurate prediction, both absorption by pigments and scattering by cells have to be considered, and because simple models, like the Lambert-Beer law, accounting only for absorption are not adapted (Leifer, 1988; Stramigioli et al., 1982), the general theory of radiative transfer must be applied to model light energy transfer in photobioreactors (Aiba, 1982; Cornet et al., 1994). Nevertheless, solving the general form of the radiative transfer equation to a finite medium such as a photobioreactor requires complex numerical tools and long calculation time. This prevents such a method from being implemented for modeling a dynamic process like a culture growth. A compromise has to be found between the simplicity and complexity described above. Cornet et al. (1995) have applied a simple two-flux model to various photobioreactor geometries where a one-dimensional approximation can be assumed. Light attenuation in the medium takes place along only one coordinate, like a rectangular photobioreactor illuminated on one or both sides, cylindrical or spherical geometry with radial illumination. This model provides an analytical solution that is useful for real-time control of a photobioreactor for example.

In this study, the two-flux approach was applied to the Chlamydomonas reinhardtii growth in a photobioreactor of torus shape. The model was applied in consideration of the light transfer problem with only a rectangular geometry illuminated on the front side with reflection on the back side, and with a validated assumption of a quasi-collimated field of radiation inside the reactor. The two-flux method requires the determination of the radiative properties for the medium (absorption and scattering coefficients, back-scattered fraction 
of light) and the mean incident light flux. Because of its crucial importance to describe light transfer as a boundary condition, two methods were compared to measure the incident light flux; an integral method using a chemical actinometer (Reinecke's salt) and a spatial integration using a plane cosine sensor. Optical properties of microalgae were determined using in vivo pigments absorption spectra data bank. Such an approach allows the two-flux method to be applied in a fully predictive manner. Only pigments content, size distribution, and shape of microalgae cultivated need to be measured. Next, applying the Lorenz-Mie theory for each microorganism considered as a pseudo-homogeneous scatterer leads to the calculation of the radiative spectral properties by a predictive mean, as already performed by Wyatt (1968) for dielectric microorganisms. Such parameters can be validated by high accuracy transmittance measurements using an integrating sphere photometer, and compared with predictive theoretical values given by a onedimensional two-flux approximation with quasi-collimated incidence. By using the spectral radiative properties in the photosynthetically active radiation (PAR), the two-flux model is then applied in the photobioreactor to determine attenuation profiles. Comparison with experimental measurements using an underwater quantum sensor finally validates the light transfer model.

It is noteworthy that the fully predictive approach proposed in this article is of prime interest in describing rigorously the radiant light transfer in processes where physiological changes are required (mineral starvations) or appear (pigments content, intracellular reserves...) during growth in batch or continuous culture conditions. In these cases where the radiative properties are being continuously modified, it is necessary to have a theoretical tool to improve their calculation, once the physiological deviations are properly characterized. Even if stable behavior occurs in the photobioreactor, the predictive way described herein avoids extremely complex experimental determinations of scattering angular properties, and then appears as a powerful approach to provide accurate models in processes involving light transfer. The highest degree of refinement is then necessary for these purposes when kinetic coupling with reified physiological or thermodynamic variables, compensation point location, photo-inhibition phenomenon, and metabolic zones identification are involved.

\section{MATERIALS AND METHODS}

\section{Culture Conditions and Characterization of Biological Medium}

\section{Photobioreactor Description}

The photobioreactor is presented in Figure 1. The main characteristic is its torus shape, the culture being circulated by the rotation of a marine impeller. The combination of such an impeller with the loop configuration of the torus geometry allows for a good mixing, without dead volume (Pruvost et al.,
2004). The light supplying device is placed in front of the photobioreactor enabling a rectangular one-dimensional treatment in describing the light transfer process.

The photobioreactor volume is $1.3 \mathrm{~L}$, the gap width of the square channel being $40 \mathrm{~mm}$ (light path), with an external diameter of the torus shape of $260 \mathrm{~mm}$ and an internal one of $180 \mathrm{~mm}$. The reactor is managed in transparent PMMA (Polymethyl methacrylate), except for the back side that is in stainless steel for reactor cooling by ambiant air blowing (fan). The marine impeller rotation speed can be ranged from 0 to $1000 \mathrm{rpm}$, and is measured using an optical tachymeter. The photobioreactor can receive a complete loop of common sensors and automations for microalga culture, namely temperature, $\mathrm{pH}$, dissolved oxygen concentration, and gas injections $\left(\mathrm{CO}_{2}\right.$ and $\left.\mathrm{N}_{2}\right)$.

The light source has been chosen to induce an homogeneous illumination at the front plane surface of the reactor. A set of 10 daylight fluorescent tubes with small diameters of $16 \mathrm{~mm}$, was used (OSRAM L13W/12-950). These tubes are placed horizontally and parallel to the front side of the reactor, with the same total height as the reactor (Fig. 1). A reflecting mirror is placed in the back of the tubes, to reflect the light toward the reactor. The tube length of $517 \mathrm{~mm}$ is greater than the torus external diameter $(260 \mathrm{~mm})$, to avoid effects of the decrease of the illumination efficiency at tubes extremities on the torus illumination. The reactor and the light source composed of the fluorescent tubes are fixed on a framework to keep them parallel (Fig. 1), that permits modification of the distance between the light source and the reactor, and thus the incident light flux. This system allows a high incident illumination on the front photobioreactor surface, up to $1000 \mu \mathrm{E} \mathrm{m}^{-2} \mathrm{~s}^{-1}$.

\section{Microorganism and Growth Conditions}

Chlamydomonas reinhardtii $137 \mathrm{c}$ is cultivated in a Sueoka high salt (HS) medium. The medium has the following composition (in g/L): $\mathrm{NH}_{4} \mathrm{Cl}, 0.5 ; \mathrm{MgSO}_{4} \cdot 7 \mathrm{H}_{2} \mathrm{O}, 0.02$; $\mathrm{CaCl}_{2} \cdot 2 \mathrm{H}_{2} \mathrm{O}, 0.01 ; \mathrm{K}_{2} \mathrm{HPO}_{4}, 1.44 ; \mathrm{KH}_{2} \mathrm{PO}_{4}, 0.72$ and $5 \mathrm{~mL}$ of Hutner's trace elements solution (Harris, 1989). The temperature is regulated at $25^{\circ} \mathrm{C}$. The $\mathrm{pH}$ is maintained at 7.0 by automatic injection of $\mathrm{CO}_{2}$.

\section{Pigment Contents, Shape, and Size Distribution}

Nine pigments are present in Chlamydomonas reinhardtii: chlorophyll-a and chlorophyll-b, which are the most abundant, and seven carotenoids (neoxanthin, loroxanthin, violaxanthin, antheraxanthin, zeaxanthin, lutein, and $\beta$ carotene) which in the following are called photoprotective carotenoids (PPC).

The pigment content was determined using a spectrophotometer. A volume $V_{1}$ of algal suspension was centrifugated $\left(13,000 \mathrm{rpm}, 2 \mathrm{~min}, 4^{\circ} \mathrm{C}\right)$ and the supernatant was evacuated. The extraction was done with a volume $V_{2}$ of $90 \%$ (vol.) acetone during $20 \mathrm{~h}$ in the dark at $4^{\circ} \mathrm{C}$. After a second centrifugation $\left(13,000 \mathrm{rpm}, 2 \mathrm{~min}, 4^{\circ} \mathrm{C}\right)$, the absorption 

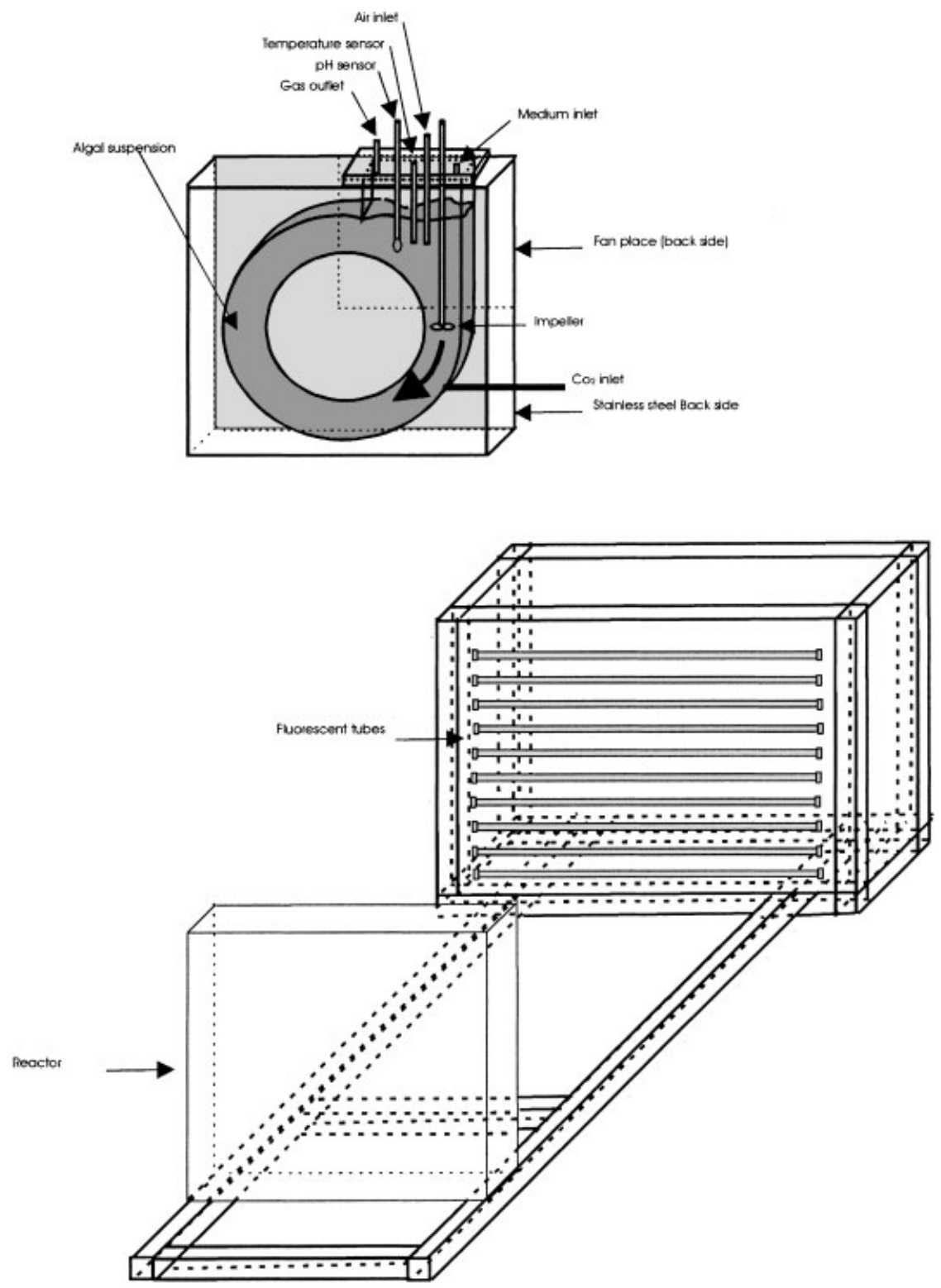

Figure 1. Schematic representation of the torus photobioreactor.

spectrum of the supernatant was collected in the range 400$750 \mathrm{~nm}$. Chlorophyll-a $\left(C_{C h l-a}\right)$, chlorophyll-b $\left(C_{C h l-b}\right)$ and photoprotective carotenoids $\left(C_{P P C}\right)$ concentrations were determined according to the following relations (Strickland and Parsons, 1968):

$$
\begin{aligned}
C_{C h l-a}= & {\left[11.6\left(\mathrm{OD}_{665}-\mathrm{OD}_{750}\right)-1.31\left(\mathrm{OD}_{645}-\mathrm{OD}_{750}\right)\right.} \\
& \left.-0.14\left(\mathrm{OD}_{630}-\mathrm{OD}_{750}\right)\right] V_{2} l^{-1} V_{1}^{-1} \\
C_{C h l-b}= & {\left[20.7\left(\mathrm{OD}_{645}-\mathrm{OD}_{750}\right)-4.34\left(\mathrm{OD}_{665}-\mathrm{OD}_{750}\right)\right.} \\
& \left.-4.42\left(\mathrm{OD}_{630}-\mathrm{OD}_{750}\right)\right] V_{2} l^{-1} V_{1}^{-1} \\
& C_{P P C}=\left[4.0\left(\mathrm{OD}_{480}-\mathrm{OD}_{750}\right)\right] V_{2} l^{-1} V_{1}^{-1}
\end{aligned}
$$

where 1 is the cell length.

The algal dry weight was determined by filtration through a predried and preweighed glass-fiber filter (Whatman GF/F).
The filter was dried at $105^{\circ} \mathrm{C}$, allowed to cool in a dessiccator, and then weighed again. The mass fraction of each pigment or group of pigments was finally determined.

Chlamydomonas reinhardtii shape and size distribution were determined by image analysis using an Olympus BX41 microscope and a video camera Kappa PS30 linked to a PCcomputer equipped with the software Image Pro Plus (Media Cybernetics $^{\circledR}$, Silver Spring, MD).

\section{Radiative-Field Measurements}

\section{Quantum Sensors}

Two types of quantum sensors were used. The incident light flux $q_{0}$ on the reactor surface was measured using a flat cosine quantum sensor (LI-190SA; LI-COR, Lincoln, NE), which measures photosynthetically active radiation (PAR) in the 
400 to $700 \mathrm{~nm}$ waveband received in a $2 \pi$ solid angle. This sensor is commonly used to determine incident light intensity in photobioreactors (Janssen et al., 2000; Sanchez Miron et al., 2003). In addition, an underwater spherical sensor was used for irradiance measurement inside the culture. This quantum sensor (US-SQS/A, Heinz Walz; LI-COR, Effeltrich, Germany) measures the photosynthetic photon fluence rate (PPFR), i.e., the light from all incoming directions $(4 \pi$ solid angle) in the PAR. The sensor diameter is $3 \mathrm{~mm}$. Because of this small sensor diameter, the total PPFR can be taken as the photosynthetic spherical irradiance (PSI, see the Appendix). The accuracy of such quantum sensors is around 5\%.

\section{Measurements of the Mean Incident Flux Using a Chemical Actinometer}

The chemical actinometer principle is based on obtaining incident light flux $q_{0}$ from the rate of a given photochemical reaction for which the quantum yield is known. Cornet et al. (1997) used Reinecke's salt $\mathrm{Cr}\left(\mathrm{NH}_{3}\right)_{2}(\mathrm{SCN})_{4}^{-}$, which presents a constant quantum yield in the range of wavelength used in photobioreactor applications (400-700 nm). Reinecke's salt irradiated in the visible range releases thiocyanate ions $\mathrm{SCN}^{-}$, that can be easily measured spectrophotometrically to determine the rate of reaction $r_{\mathrm{SCN}-}$. Cornet et al. (1997) proposed to use the following relation for a rectangular reactor illuminated on one side and operating in a physical limitation by light (all the photons entering the medium are absorbed):

$$
q_{0}=r_{\mathrm{SCN}^{-}} \frac{L}{\Phi}
$$

where $L$ is the light path length (equal to photobioreactor depth $L=40 \mathrm{~mm}$ ) and $\Phi$ the quantum yield of conversion.

The experiment can be performed for $\mathrm{pH}$ values ranged between 2 and 14 with the same accuracy, but the quantum yield of conversion is $\mathrm{pH}$ dependent, except between $\mathrm{pH} 2$ and $\mathrm{pH} 5$ where it is constant $\left(\Phi=0.29 \mathrm{~mol}_{\mathrm{SCN}-}\right.$. Einstein $^{-1}$; Cornet et al., 1997). The experiment was thus performed at $\mathrm{pH} 3.50$.

For this measurement, the stainless steel back side of the reactor was replaced by a black one, thus enabling use of Equation (4) (one side illumination without back reflection).

The actinometer was continuously stirred and temperature was kept at $23^{\circ} \mathrm{C}$. Samples were taken at regular intervals and treated with 3 volumes of iron reagent and 20 volumes of distilled water. The iron reagent was $0.1 \mathrm{~mol} / \mathrm{L}$ $\mathrm{Fe}\left(\mathrm{NO}_{3}\right)_{3}\left(\mathrm{H}_{2} \mathrm{O}\right)_{9}$ in a $0.5 \mathrm{~mol} / \mathrm{L} \mathrm{HClO}_{4}$ solution. Optical density was next measured in a spectrophotometer (Miltonroy Genisis 5) at $450 \mathrm{~nm}$, with nonirradiated solution as a blank. Experiment duration was between 2 and $3 \mathrm{~h}$.

\section{Measurement of Attenuation Profiles in the Photobioreactor}

To obtain experimental light attenuation in the torus photobioreactor, a batch culture of Chlamydomonas rein- hardtii was conducted with growth conditions previously described. After 7 to 10 days, the culture was stopped. The biomass dry weight was then determined and experimental measurements of irradiance $G$ along the depth of the photobioreactor were carried out. The 3-mm diameter underwater spherical sensor was introduced in the culture by the top of the photobioreactor. Nine values were taken uniformly along the $40 \mathrm{~mm}$ of culture depth. Measurements were repeated for different biomass concentrations obtained by successive dilutions with fresh medium.

\section{True Transmittance Measurements}

As the two sensors are sensitive in the 400 to $700 \mathrm{~nm}$ waveband (PAR), transmittances were determined in this range using an optical bench developed by SAFAS $^{\circledR}$ (Monaco). This bench is composed of three units:

(1) A $150-\mathrm{mm}$ diameter integrating sphere (Labsphere ${ }^{\circledR}$, North Sutton, NH) that offers two main advantages: first, its large size minimizes sphere errors; second, it is internally covered with SPECTRALON ${ }^{\circledR}$ - a thermoplastic material reflecting $99 \%$ of incident light from 400 to $1500 \mathrm{~nm}$.

(2) A spectrofluorometer Flx $\left(\mathrm{SAFAS}^{\circledR}\right)$ linked to the sphere by means of optical fibers. A Xenon lamp produces the incident beam with an important power which is required for the $150-\mathrm{mm}$ diameter sphere that attenuates the incident light by 5 orders of magnitude.

(3) A PC-computer equipped with an acquisition card and the SAFAS ${ }^{\circledR}$ Spectro2000 software.

\section{PREDICTIVE LIGHT TRANSFER MODELING}

As previously discussed, the radiative field calculation in photobioreactors is of critical relevance in the process modeling. Various models have been applied, including Lambert-Beer law which is of low accuracy in photobioreactors because of the neglection of light scattered by cells in the medium (Aiba, 1982; Cornet et al., 1992, 1994, 1995; Spadoni et al., 1978). For a proper description of the light transfer, the radiative transfer equation (RTE) must be solved. In the case of the torus geometry reactor, because the incident light is perpendicular to the front side of the reactor, the one-dimensional approximation is relevant and the specific intensity $I_{\lambda}$ depends only on the depth of culture (z-abscissa), so the RTE becomes (Chandrasekhar, 1960):

$$
-\cos (\theta) \frac{d I_{\lambda}}{d z}=\left(a_{\lambda}+s_{\lambda}\right) I_{\lambda}-\frac{s_{\lambda}}{2} \int_{0}^{\pi} I_{\lambda} p_{\lambda}\left(\theta, \theta^{\prime}\right) \sin \left(\theta^{\prime}\right) d \theta^{\prime}
$$

where $I_{\lambda}$ is the spectral specific intensity, $a_{\lambda}$ and $s_{\lambda}$ are the spectral volumetric absorption and scattering coefficients, and $p_{\lambda}\left(\theta, \theta^{\prime}\right)$ the spectral phase function for scattering.

If the coefficients $a_{\lambda}, s_{\lambda}$, and $p_{\lambda}\left(\theta, \theta^{\prime}\right)$ can be obtained theoretically, Equation (5) is fully predictive. 


\section{Predictive Determination of Culture Optical and Radiative Properties}

\section{Wavelength Dependent Properties}

The optical properties necessary to formulate the radiative transfer model can be calculated from the Lorenz-Mie theory, knowing the refractive index $n_{m}$ of the surrounding medium (water in a first approximation), and the complex refractive index of the particle $m=n \pm i \kappa_{\lambda}$, where $n$ is the real part of the refractive index for the particle, mainly responsible for scattering, and $\kappa_{\lambda}$ is the imaginary part of the refractive index responsible for absorption. Therefore, we consider in the following that $n$ is not a spectral quantity whereas $\kappa_{\lambda}$ strongly varies with the wavelength according to the pigment composition of the cell.

The real part $n$ of the refractive index can be determined either experimentally or theoretically (Bohren and Huffman, 1983). The theoretical method requires knowledge of the elemental refractive index and the volume fraction of each component of the cell material (cell membrane, cytoplasm, mitochondria...). Experimental determination requires the measurement of the transmittance at a nonabsorbed wavelength (between 790 and $830 \mathrm{~nm}$ for Chlamydomonas reinhardtii) with an integrating sphere photometer.

The imaginary part $\kappa_{\lambda}$ of the refractive index depends on the pigment content. In this work, we propose a new predictive approach to calculate this wavelength dependent coefficient from the knowledge of the pigment content.

The predictive determination of the imaginary part of the refractive index $\kappa_{\lambda}$ is based on the electromagnetic theory which gives the following relation between $\kappa_{\lambda}$ and the volumetric coefficient of the cell material $a_{c m}$ (Bohren and Huffman, 1983):

$$
\kappa_{\lambda}=\frac{a_{c m} \lambda}{4 \pi}=\frac{\lambda}{4 \pi} \sum_{i=1}^{N} E a_{p i g . i}(\lambda) \cdot C_{p i g . i}
$$

where $E a_{\text {pig.i } i}(\lambda)$ is the in vivo spectral mass absorption coefficient of pigment $i$, and $C_{p i g . i}$, the concentration of pigment $i$ in the cell (with a total of $N$ pigment species).

Knowing the mass fraction of each pigment $\left(w_{\text {pig. }}\right)$, the density of the dry material and the in vivo volume fraction of water in the cell $\left(x_{w}\right)$, we obtain the following relation giving the value of the imaginary part of the complex refractive index by convolution:

$$
\kappa_{\lambda}=\frac{\lambda}{4 \pi} \rho_{d m} \frac{1-x_{w}}{x_{w}} \sum_{i=1}^{N} E a_{p i g . i}(\lambda) \cdot w_{p i g . i}
$$

where

$$
x_{w}=1-\frac{C_{x}}{N_{p}} \frac{1}{V_{32}} \frac{1}{\rho_{d m}}
$$

in which $C_{x}$ is the biomass dry weight concentration, $N_{p}$ the cell number density and $V_{32}$ the mean (Sauter) particle volume taking into account the given size distribution.
To use this predictive method, the spectral mass absorption coefficient for all the pigments must be known in in vivo conditions. Very little information is available in the relevant literature on this subject, nevertheless Bidigare et al. (1990) estimated the in vivo specific absorption coefficient for the major algal pigments groups among which were chlorophyll a, chlorophyll b, and photoprotective carotenoids (Fig. 2).

Knowing the particle refractive index (real and imaginary parts), the mean equivalent diameter (the particle is considered as pseudo-spherical) and the size distribution of the considered microalga, the predictive calculation of the radiative properties can be performed from the LorenzMie theory for spherical particles, using a numerical fortran code adapted from Bohren and Huffman (1983). This code enables the calculation of the absorption and scattering cross-sections $\left(C_{A B S}\right.$ and $\left.C_{S C A}\right)$ and the phase function $p\left(\theta, \theta^{\prime}\right)$ for a given size parameter $x=\pi d_{p} / \lambda$ of the considered microorganism with a mean diameter $d_{p}$. This last angular function serves as a basic tool used in what follows to compute the backward scattering fraction $b$. The mass and volumetric absorption and scattering coefficients $\left(E a_{\lambda}, a_{\lambda}\right.$, $E s_{\lambda}$, and $s_{\lambda}$ ) can then be calculated from their respective cross-sections by the following equations:

$$
E a_{\lambda}=\frac{C_{A B S}(\lambda)}{V_{32} \rho_{d m}\left(1-x_{w}\right)}, E s_{\lambda}=\frac{C_{S C A}(\lambda)}{V_{32} \rho_{d m}\left(1-x_{w}\right)}
$$

and

$$
a_{\lambda}=E a_{\lambda} C_{X}, s_{\lambda}=E s_{\lambda} C_{X}
$$

leading, together with the phase function for scattering, to the required spectral radiative properties by an entirely predictive method.

\section{Mean Properties in Wavelength}

Many modeling approaches in photobioreactors use mean coefficients in wavelength to avoid tedious spectral computation and averaging of radiant variables. In this case, what

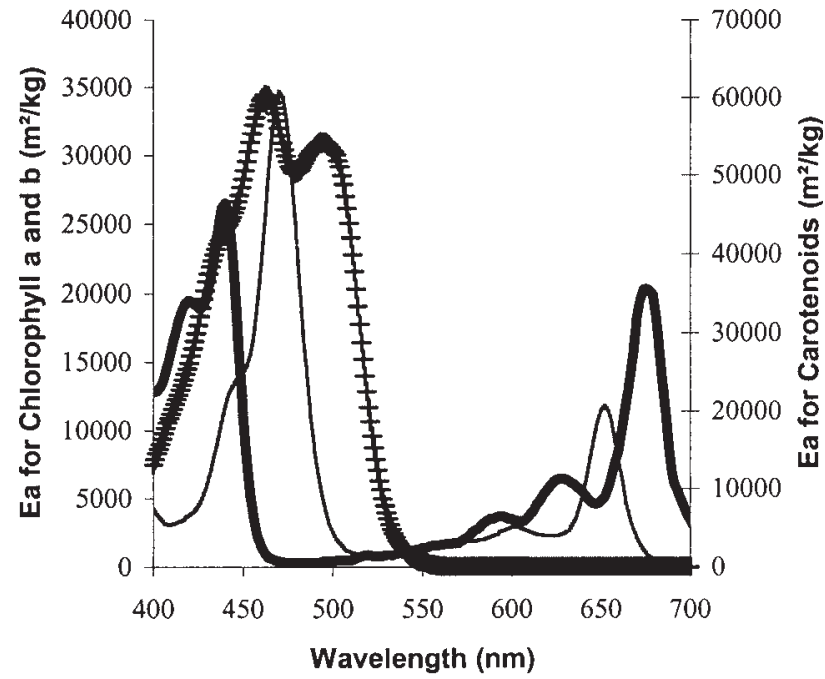

Figure 2. Data bank of in vivo absorption coefficients of pure pigments ( $\sim$ chlorophyll a, - chlorophyll b, +++ carotenoids). Data are from Bidigare et al. (1990). 
the best method is for obtaining mean values on the visible spectrum from spectral elemental data is open to debate. Certainly, a simple averaging of the spectral imaginary part of the refractive index $\kappa_{\lambda}$ between the minimum $\lambda_{m}$ and the maximum $\lambda_{m}$ wavelength range of interest:

$$
\kappa *=\frac{\int_{\lambda_{m}}^{\lambda_{M}} \kappa_{\lambda} d \lambda}{\Delta \lambda}
$$

is not sufficient, although it is the first stage in the search for a mean coefficient, as according to electromagnetic theory the absorption efficiency $Q_{A B S}$ does not vary linearly with the wavelength. Rigorously, the dimensionless function $\mathrm{Q}_{\lambda}^{*}$ that allows for this nonlinearity is given by the Lorenz-Mie theory (Morel and Bricaud, 1981; Van de Hulst, 1981):

$$
\mathrm{Q}_{\lambda}^{*}=\frac{3}{2} \frac{Q_{A B S}(\xi)}{\xi}
$$

where the particle optical thickness $\xi$ is given by:

$$
\xi=4 x \kappa_{\lambda} / n_{m}
$$

$x$ being the well-known size parameter previously defined (Bohren and Huffman, 1983). In the case where $\xi=0$ (i.e., the particle does not exist), the $Q_{\lambda}^{*}$ function is equal to one, corresponding to the molecular absorption of the pigments themselves, and there is no reason to modify the averaged value given by Equation (13). On the contrary, if the parameter $\xi$ increases (which is the case for any particle), the $\mathrm{Q}_{\lambda}^{*}$ function decreases, taking into account the so-called packaging effect of the Lorenz-Mie theory (Morel and Bricaud, 1981). Because this function is a spectral quantity, it is then possible to average it on the chosen spectrum to obtain a better estimate of the mean imaginary part of the refractive index for the microorganism. For a sphere, and applying the anomalous diffraction proposed by Van de Hulst (1981), which is well suited to microorganisms, the $Q_{\lambda}^{*}$ function can be developed as a $\xi$ variable function by:

$$
Q_{\lambda}^{*}=\frac{3}{2 \xi}\left[1+\frac{2 \mathrm{e}^{-\xi}}{\xi}+2\left(\frac{\mathrm{e}^{-\xi}-1}{\xi^{2}}\right)\right]
$$

enabling us to average this spectral function over the considered domain of wavelength $\Delta \lambda$, corresponding to the $\Delta \xi$ domain between $\xi_{m}$ and $\xi_{M}$ from:

$$
Q^{*}=\frac{\int_{\xi_{m}}^{\xi_{M}} Q^{*}(\xi) d \xi}{Q^{*}\left(\xi^{*}\right) \Delta \xi}
$$

$\xi *$ being obtained by Equation (15) using $\kappa^{*}$ [Equation (13)], then:

$$
\begin{aligned}
Q^{*}= & \frac{3}{2 \Delta \xi Q^{*}\left(\xi^{*}\right)}\left[\ln \left(\frac{\xi_{M}}{\xi_{m}}\right)+\frac{1}{\xi_{M}^{2}}-\frac{1}{\xi_{m}^{2}}+\frac{1}{\xi_{m} e^{\xi_{m}}}\left(1+\frac{1}{\xi_{m}}\right)\right. \\
& \left.-\frac{1}{\xi_{M} e^{\xi_{M}}}\left(1+\frac{1}{\xi_{\mathrm{M}}}\right)+\operatorname{Ei}\left(1, \xi_{M}\right)-\operatorname{Ei}\left(1, \xi_{m}\right)\right]
\end{aligned}
$$

where $\operatorname{Ei}(n, x)$ is the exponential integral $\operatorname{Ei}(n, x)=\int_{1}^{\infty} \frac{e^{-x t}}{t^{n}} d t$
Table I. Characteristics and basic optical properties of Chlamydomonas reinhardtii $137 \mathrm{c}$ cultivated in the Sueoka high salt medium.

\begin{tabular}{lc}
\hline Mean diameter $(\mathrm{m})$ & $7.9810^{-6}$ \\
\hline Standard deviation for the log-normal size distribution & 1.17 \\
Mean effective volume $V_{32}\left(\mathrm{~m}^{3}\right)$ & $3.1910^{-16}$ \\
Pigment content $(\% \mathrm{w})$ & \\
Chlorophyll-a & 1.4 \\
Chlorophyll-b & 0.7 \\
Photoprotective carotenoids $(\mathrm{PPC})$ & 0.45 \\
Water volume fraction $x_{w}$ & 0.78 \\
Refractive index (real part) $n$ & 1.527 \\
Mean refractive index (imaginary part) $\kappa$ & 0.00327 \\
$Q^{*}$ & 0.96 \\
\hline
\end{tabular}

Equation (18) with Equation (13) is then a basic relation giving the best assessment of the mean imaginary part of the refractive index in wavelength from (Table I):

$$
\kappa=\kappa^{*} Q^{*}
$$

This value, with the constant real part of the refractive index $n$, can be used with the Lorenz-Mie theory to compute the mean absorption and scattering coefficients, together with the mean phase function for scattering over a considered range of wavelength. In this case, from the mean value of $\kappa$ (Table I) and from Equations (9-12), the coefficients are the following for Chlamydomonas reinhardtii in the PAR:

$$
E a=172 \mathrm{~m}^{2} / \mathrm{kg} ; E s=868 \mathrm{~m}^{2} / \mathrm{kg} ; b=0.01728 .
$$

\section{The Two-Flux Method to Solve the Radiative Transfer Equation}

Different numerical methods exist to solve exactly Equation (5) (Siegel and Howell, 2002), but they require long calculation times. Simple solutions are often preferred, especially to be implemented in model-based predictive control of photobioreactors or to be used in the complex description of the kinetic coupling. An interesting model for radiant light energy transfer in a culture medium derives from the generalized two-flux approximation (Mengüç and Viskanta, 1983). This approach has already been used for photobioreactor's modeling (Cornet et al., 1998; Cornet and Albiol, 2000) with experimental and approximative determination of the radiative properties of the medium. It considers that the scattered radiation is confined to the forward and backward directions. The form of the resulting system depends on the assumption made for the positive and negative radiation fields involving specific intensities independent of the polar angle $\theta$, i.e., diffuse or collimated. Even if it has been recently used by authors (Cornet et al., 2001, 2003), the hypothesis of a diffuse radiation field is not fulfilled for most artificial lightening devices used for photobioreactors, that are better represented by a quasicollimated incident radiation field.

Using a collimated assumption for the incident light flux making an angle $\theta_{c}$ with the normal of the medium, 
Hottel and Sarofim (1967) obtain the following system of differential equations in place of the exact RTE [Equation (5)]:

$$
\left\{\begin{array}{l}
\cos \left(\theta_{c}\right) \frac{d I_{\lambda}^{+}}{d \tau_{\lambda}}=\left(\omega_{\lambda}-1\right) I_{\lambda}^{+}-\omega_{\lambda} b_{\lambda}\left(I_{\lambda}^{+}-I_{\lambda}^{-}\right) \\
\cos \left(\theta_{c}\right) \frac{d I_{\lambda}^{-}}{d \tau_{\lambda}}=\left(1-\omega_{\lambda}\right) I_{\lambda}^{-}-\omega_{\lambda} b_{\lambda}\left(I_{\lambda}^{+}-I_{\lambda}^{-}\right)
\end{array}\right.
$$

where $\tau_{\lambda}$ is the optical thickness, i.e.: $\tau_{\lambda}=\left(E a_{\lambda}+E s_{\lambda}\right) C_{x} L$; $\omega_{\lambda}$ the albedo for single scattering, i.e.: $\omega_{\lambda}=E s_{\lambda}$ / $\left(E a_{\lambda}+E s_{\lambda}\right)$, and $b_{\lambda}$ is the backward scattering fraction defined as:

$$
b_{\lambda}=\frac{1}{2} \int_{\pi / 2}^{\pi} p_{\lambda}\left(\theta, \theta^{\prime}\right) \sin (\theta) d \theta
$$

validation of the radiative properties of Chlamydomonas reinhardtii.

Nevertheless, this method can be extended for the considered photobioreactor in this study by giving proper boundary conditions for the system of differential equations (I), that are illumination on the front side with reflection on the back side:

$$
\begin{cases}z=0, & I_{\lambda}^{+}=I_{\lambda, 0} \cos \left(\theta_{c}\right)=q_{\lambda, 0} \\ z=L, & I_{\lambda}^{-}=\rho I_{\lambda}^{+}\end{cases}
$$

where $I_{\lambda, 0}$ is the incident quasi-collimated intensity, $\rho$ the reflection coefficient of the stainless steel at the rear of the reactor, and $L$ the light path length (reactor depth).

Considering these boundary conditions with the quasicollimated hypothesis in the two-flux model (I), analytical expression for the spectral irradiance $\left(G_{\lambda}\right)$ attenuation is obtained:

$$
\begin{aligned}
& \frac{G_{\lambda}(z)}{q_{\lambda, 0}} \\
& \quad=2 \sec \left(\theta_{c}\right) \frac{\left[\rho\left(1+\alpha_{\lambda}\right) \exp \left(-\delta_{\lambda} L\right)-\left(1-\alpha_{\lambda}\right) \exp \left(-\delta_{\lambda} L\right)\right] \exp \left(\delta_{\lambda} z\right)+\left[\left(1+\alpha_{\lambda}\right) \exp \left(\delta_{\lambda} L\right)-\rho\left(1-\alpha_{\lambda}\right) \exp \left(\delta_{\lambda} L\right)\right] \exp \left(-\delta_{\lambda} z\right)}{\left(1+\alpha_{\lambda}\right)^{2} \exp \left(\delta_{\lambda} L\right)-\left(1-\alpha_{\lambda}\right)^{2} \exp \left(-\delta_{\lambda} L\right)-\rho\left(1-\alpha_{\lambda}^{2}\right) \exp \left(\delta_{\lambda} L\right)+\rho\left(1-\alpha_{\lambda}^{2}\right) \exp \left(-\delta_{\lambda} L\right)}
\end{aligned}
$$

These authors solved the system for a bounded medium with no radiation entering at the rear surface, and established the expressions for reflectance and transmittance at boundaries. The complete resolution of system (I) with notations more convenient to photobioreactor applications is similar to the one which was carried out by Cornet et al. (1995) in the case of a diffuse incidence. Thus, the expression of transmittance $\left(T_{\lambda}\right)$, useful for exploiting experimental results obtained with the integrating sphere photometer, is given by:

$$
T_{\lambda}=\frac{4 \alpha_{\lambda}}{\left(1+\alpha_{\lambda}\right)^{2} \exp \left[\delta_{\lambda} L\right]-\left(1-\alpha_{\lambda}\right)^{2} \exp \left[-\delta_{\lambda} L\right]}
$$

where,

$$
\delta_{\lambda}=C_{X} \sec \left(\theta_{c}\right) \sqrt{E a_{\lambda}\left(E a_{\lambda}+2 b_{\lambda} E s_{\lambda}\right)}
$$

is the two-flux extinction coefficient, and

$$
\alpha_{\lambda}=\sqrt{\frac{E a_{\lambda}}{E a_{\lambda}+2 b_{\lambda} E s_{\lambda}}}
$$

ranging between 0 (perfect scattering medium) and 1 (perfect absorptive medium) is the linear scattering modulus which quantifies the respective importance of absorption and scattering phenomena, but taking into account the phase function for the microorganisms. Those Equations (21)-(23) are of particular interest in this study because they apply especially in an integrating sphere photometer and will serve later as a theoretical basis for the where $\delta_{\lambda}$ and $\alpha_{\lambda}$ are defined as in Equations (22) and (23). This spectral equation applies for any wavelength in the PAR, but may be used for the total visible radiation in defining properly the mean parameters $E a, E s$, and $b$ as discussed above. A special emphasis has been paid on Equations (21)(24) for a quasi-collimated incidence making an angle $\theta_{c}$ with the normal because, combining these equations with the same approach for a diffuse incidence (Cornet et al., 1995) provides an excellent basis for modeling light transfer in solar photobioreactors with direct illumination by splitting the radiation field in a constant diffuse part and a polar time dependent collimated part.

\section{APPLICATION AND EXPERIMENTAL VALIDATION OF THE PREDICTIVE MODEL}

\section{Validation of the Predictive Determination of Radiative Properties for Chlamydomonas reinhardtii}

The size characteristics and the experimental pigment contents for Chlamydomonas reinhardtii cultivated in the Sueoka high salt medium are given in Table I. The corresponding values of imaginary refractive index are then calculated [Equation (7)] and plotted in Figure 3 for the 400 to $700 \mathrm{~nm}$ waveband (PAR). It can be noted that these calculated values for $\kappa_{\lambda}$ display a very good agreement for peaks and valleys with the classical absorption spectrum obtained from direct measurements of intact Chlamydomonas reinhardtii living samples. Moreover, the image analysis 


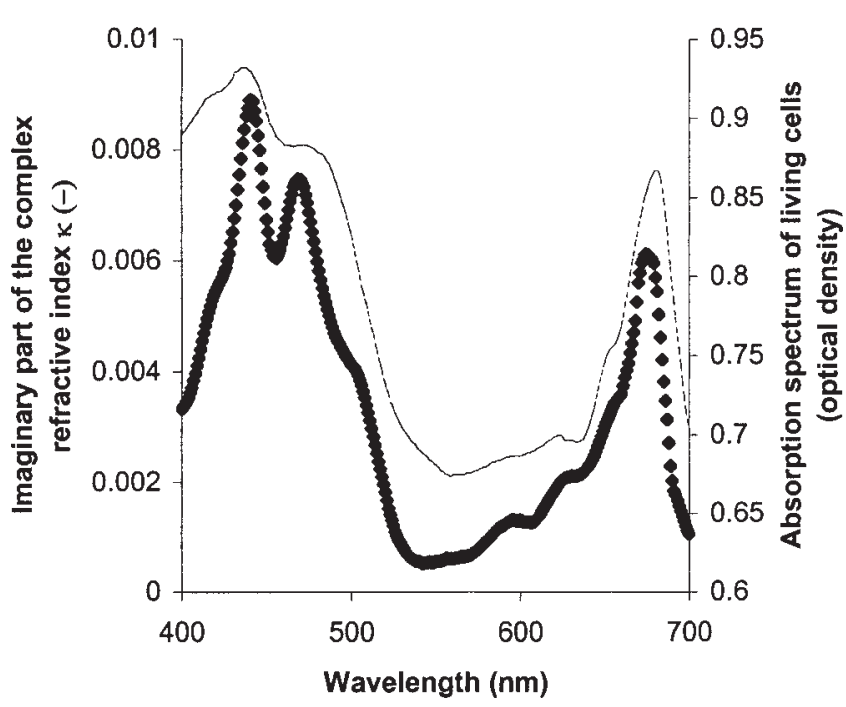

Figure 3. Imaginary part of the refractive index for Chlamydomonas reinhardtii calculated from data banks and mass fraction of pigments [equation (7)] ( $\diamond$ ) and absorption spectrum of living cells (-).

shows that Chlamydomonas reinhardtii can be considered as a spherical equivalent particle, with a diameter of $7.98 \mu \mathrm{m}$ and a size distribution of lognormal type with a standard deviation of 1.17 (Table I). The real part of the complex refractive index is determined at $820 \mathrm{~nm}$ which is a nonabsorbed wavelength for Chlamydomonas reinhardtii using the integrating sphere photometer and is equal to 1.527 . The relative real part of the refractive index $n / n_{m}$ is then assumed to be constant on the visible spectrum (PAR). This assumption, with the previous information, enables us to determine the so-called radiative properties of Chlamydomonas reinhardtii, applying the Lorenz-Mie theory (Bohren and Huffman, 1983). The resulting values for spectral mass absorption and scattering coefficients, with back-scattered fractions are depicted in Figure 4. These values give an albedo for single scattering $\omega_{\lambda}$ in the range $0.65-0.95$ (results not shown), clearly demonstrating that scattering by particles in the turbid medium is the dominant process. Nevertheless, this conclusion must be moderated because the phase function being strongly peaked in the forward direction, the resulting backscattered fractions are quite low $(1-3 \%)$.

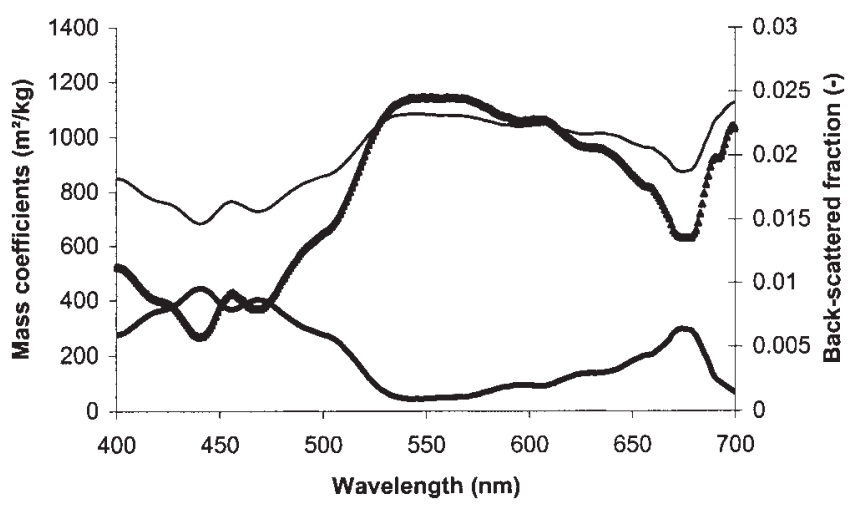

Figure 4. Spectrum of predicted back-scattered fraction (A৯), mass absorption ( - ) and scattering (-) coefficients $E a(\lambda)$ and $E s(\lambda)$, for Chlamydomonas reinhardtii.

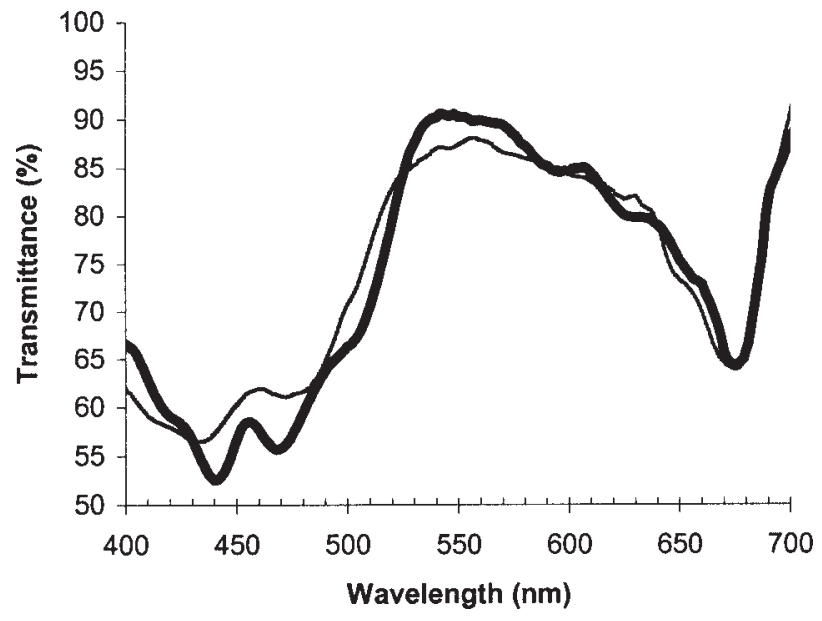

Figure 5. Comparison of true transmittances obtained by the two-flux model with predictive radiative properties (equations $7-12$ and 20-23) (-) and experimental ones measured with the integrating sphere photometer for a biomass concentration of $0.15 \mathrm{~g} / \mathrm{L}$ and a light path length of $1 \mathrm{~cm}(-)$.

Finally, in Figure 5, experimental transmittances obtained with the integrating sphere photometer are compared to the theoretical ones calculated using the two-flux model applied with predicted radiative properties [Equation (21) and Fig. 4]. The evolution of transmittances in the range of photosynthetically active radiation appears in particularly good agreement (mean deviation of 3\%) taking into account that the two-flux approximation corresponds to a differential discrete ordinates method of first order only (Siegel and Howell, 2002). These results confirm the validity of the predictive approach to calculate radiative properties for Chlamydomonas reinhardtii cultures from only the optical and size characteristics of the microorganism.

\section{Determination of the Incident Light Flux $\boldsymbol{q}_{0}$}

To calculate the radiative transfer profile in the photobioreactor, the incident flux $q_{0}$ first needs to be determined [Equation (24)]. The incident flux $q_{0}$ was measured using an actinometric method and a plane cosine quantum sensor. The actinometric reaction being sensitive to light incoming from all directions, the comparison with surface measurements using the plane sensor verifies if the culture medium in the photobioreactor can be assumed to be only illuminated from the front (no parasitical light coming from the reactor sides).

The results presented in Table II were first obtained with a distance of $80 \mathrm{~mm}$ between light source and the reactor. With the actinometric method [Equation (4)], the mean incident radiant energy flux $q_{0}$ calculated is equal to $440 \mu \mathrm{E} \mathrm{m}^{-2} \mathrm{~s}^{-1}$ (Table II). By averaging plane sensor measurements for eight different locations on the reactor front side (Fig. 6), an incident light flux of $310 \mu \mathrm{E} \mathrm{m}^{-2} \mathrm{~s}^{-1}$ is obtained.

The discrepancies obtained between both methods show that the total incident light effectively received by the culture is $50 \%$ higher than the one-dimensional quasi-collimated expected light on the front of the reactor, despite a lightening set-up that would allow a uniform front light distribution 
Table II. Incident light flux densities or irradiances in $\mu \mathrm{E} \cdot \mathrm{m}^{-2} \cdot \mathrm{s}^{-1}$ obtained with the two sensors and the actinometric reaction for the three configurations tested. For the actinometric method, the hypothesis of quasicollimated incidence implies that incident flux equal irradiance $\left(q_{0}=G_{0}\right)$.

\begin{tabular}{lccc}
\hline & $\begin{array}{c}\text { Cosine } \\
\text { plane sensor } \\
\text { (Flux density) }\end{array}$ & $\begin{array}{c}\text { Spherical } \\
\text { sensor } \\
\text { (Spherical } \\
\text { irradiance) }\end{array}$ & $\begin{array}{c}\text { Actinometric } \\
\text { reaction } \\
\text { (Irradiance } \\
\text { or flux) }\end{array}$ \\
\hline $80 \mathrm{~mm}$ without mask & $310 \pm 20$ & $440 \pm 30$ & $440 \pm 30$ \\
$80 \mathrm{~mm}$ with masks & $280 \pm 20$ & $330 \pm 20$ & $320 \pm 20$ \\
$250 \mathrm{~mm}$ with masks & $130 \pm 10$ & $140 \pm 10$ & $140 \pm 10$ \\
\hline
\end{tabular}

(plane surface, fluorescent tubes parallel to the front face of the reactor). To verify this assumption, an additional experiment is done with the spherical quantum sensor which integrates light incoming from all directions $(4 \pi$ solid angle), giving a spherical irradiance in first approximation (see Appendix). Forty-eight values uniformly distributed on the illuminated side (Fig. 6) were averaged. The corresponding incident flux obtained was $440 \mu \mathrm{E} \mathrm{m}^{-2} \mathrm{~s}^{-1}$ (Table II) that is in excellent agreement with the actinometric method. This confirms that the inside volume of the reactor receives light from other sides due to a possible refraction of the PMMA walls of the photobioreactor, impairing the rectangular onedimensional hypothesis for the light transfer problem. This demonstrates that attention must be paid when using such a flat cosine sensor to evaluate incident illumination, especially when rigorous modeling of the process is envisaged, and that a comparison between cosine sensor for flux and spherical sensor for irradiance should be always envisaged to properly characterize the incident field of radiation.

Considering previous results, the photobioreactor was then modified to reduce the parasitical side illumination to fulfil the one-dimensional approximation (light attenuation along only one coordinate). Black masks were placed on the photobioreactor surface to restrict illumination to the torus shape.

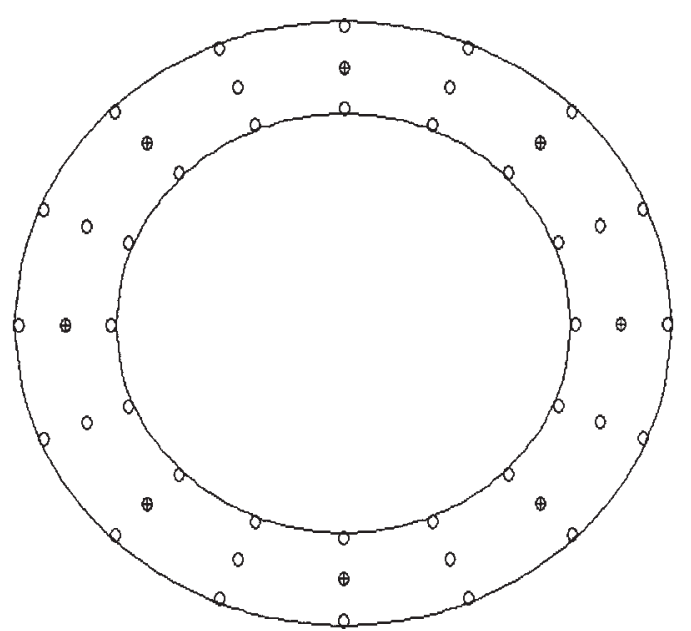

Figure 6. Locations of surface measurements using quantum sensors for flux density or irradiance determination ( + : plane sensor measurements, $\mathrm{o}$ : spherical sensor measurements).
The results obtained with this modified set-up are presented in Table II for two spacings between light source and the reactor ( 80 and $250 \mathrm{~mm}$ ). Values from the two sensors are very close, that shows the efficiency of masks to prevent the parasitical sides illumination. It appears that the experimental values obtained with spherical sensor and actinometer are the same, whereas the values of the flat cosine sensor are slightly lower. For a perfectly collimated beam, these values should be the same, and for a diffuse irradiance, they should differ by a factor 2 . As a consequence, one can conclude that the quasi-collimated assumption for the incident light flux seems very good in all cases, but it is better for a higher distance (lower light flux).

\section{Application of the Light Transfer Model to the Torus Photobioreactor}

As previously discussed, two approaches are available to investigate light attenuation into the photobioreactor; by working with the wavelength-dependent mass coefficients $E a(\lambda), E s(\lambda)$, and back-scattered fraction $b(\lambda)$, then averaging spectral intensities in the PAR, or by using first direct mean values of radiative properties on the considered range of wavelength.

Simulations and experimental measurements are done for three different biomass dry-weight concentrations: 0.09, 0.25 , and $0.49 \mathrm{~g} / \mathrm{L}$ (respectively, optical thicknesses: 4,10 , and 20). In each case, the theoretical profiles were calculated by the fully predictive model proposed [using Equation (24) with a normal incidence, i.e., $\cos \left(\theta_{c}\right)=1$ ], either with mean or spectral coefficients (from the data in Fig. 4). In this latter case, it is necessary to define a method in the calculation of the spectral irradiance $G_{\lambda}$ to use Equation (24) properly, whereas there is no ambiguity concerning the definitions of the mean irradiance $G$ and mean incident light flux $q_{0}$ in the PAR as discussed above. Assuming an homogeneous incident spectrum in the PAR for the lamps, the spectral incident light flux is then easily calculated from $q_{0}(\lambda)=q_{0} / N$ where $N$ stands for the 300 wavelengths corresponding to spectral radiative properties calculated for each nanometer in the visible spectrum. Finally, the considered spectral irradiance $G_{\lambda}$ on a wavelength interval of $1 \mathrm{~nm}$ is then related to the averaged irradiance $G_{\Sigma}$ in the PAR by the following summation:

$$
G_{\Sigma}(z)=\sum_{\lambda_{i}=400 \mathrm{~nm}}^{\lambda_{i}=700 \mathrm{~nm}} G_{\lambda_{i}}(z)
$$

which may then be directly compared with the mean irradiance $G$.

Figure 7 displays the results obtained for the three biomass concentrations considered in the photobioreactor. It clearly appears that the proposed predictive two-flux model agrees very well with experimental measurements of irradiance inside the reactor. Besides, the use of spectral radiative properties improves the prediction in the depth of the culture, especially when increasing the biomass concentration towards the limit of a physical limitation by $\operatorname{light}\left(C_{X}=0.5 \mathrm{~g} / \mathrm{L}\right.$, 
a

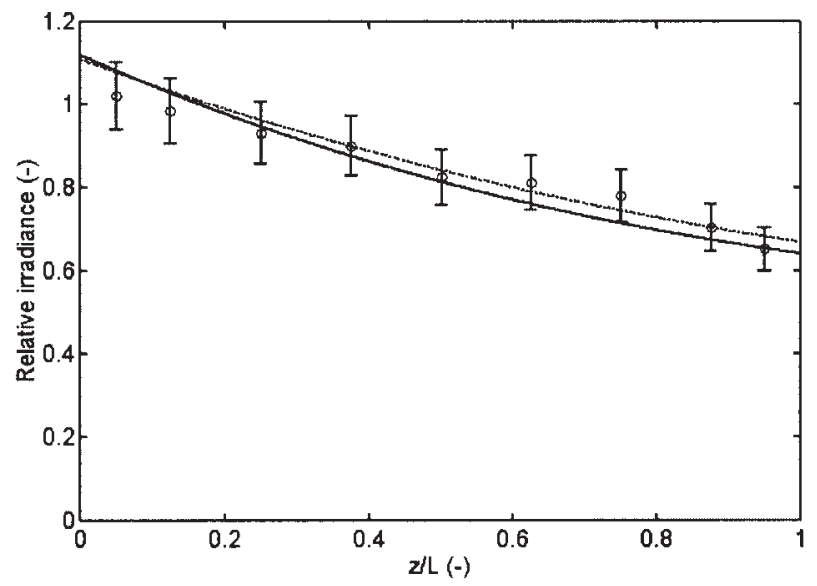

b

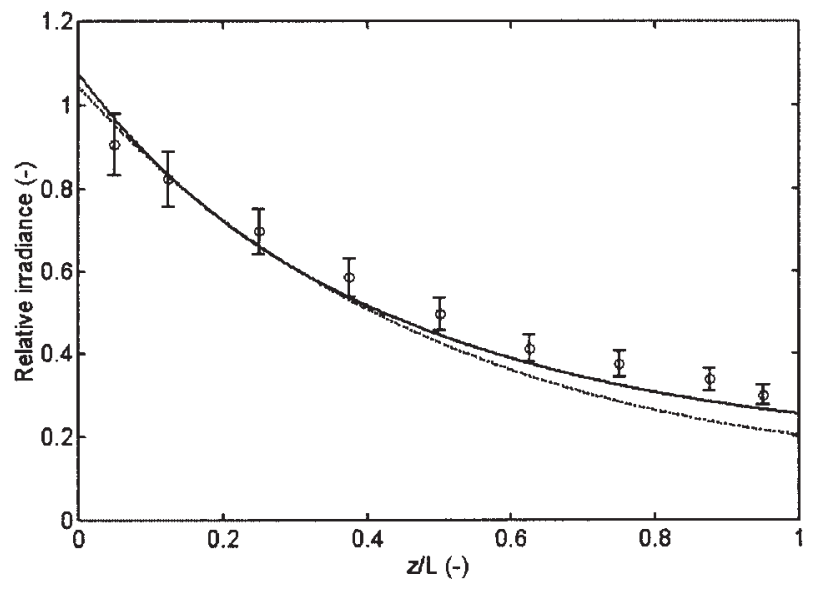

C

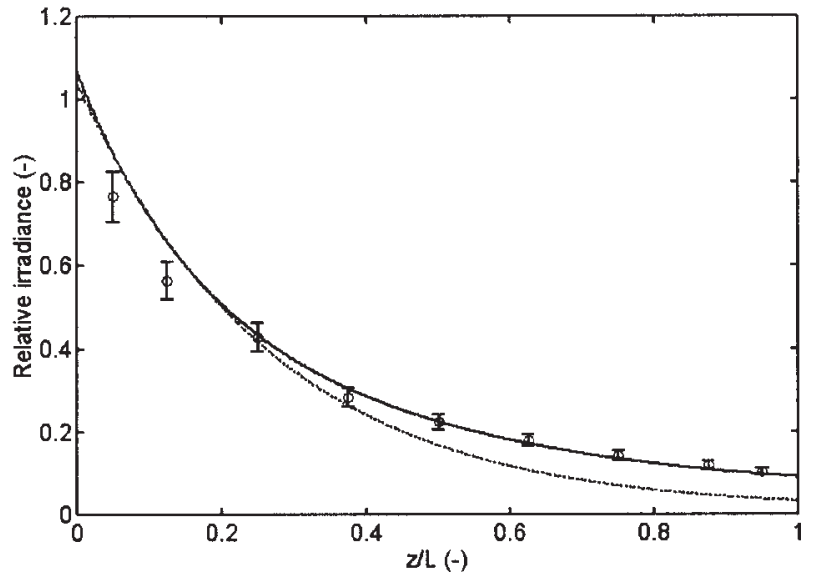

Figure 7. $(a-c)$ Comparison between attenuation profiles in the photobioreactor and experimental irradiances measured with a spherical quantum sensor of $3 \mathrm{~mm}$ diameter (solid line: $\frac{G_{\Sigma}}{q_{0}}$, dotted line: $\frac{G}{q_{0}}, \bigcirc$ : experimental values). (a) $C_{X}=0.09 \mathrm{~g} / \mathrm{L}$ or $\tau=4$, (b) $C_{X}=0.25 \mathrm{~g} / \mathrm{L}$ or $\tau=10$ (c) $C_{X}=0.49 \mathrm{~g} / \mathrm{L}$ or $\left.\tau=20\right)$.

$\tau=20$ ), obtained at the appearance of the photosynthesis compensation point at the rear of the reactor. This is explained by results presented in Figure 8, where evolution of the light spectrum along the depth of the culture is calculated with wavelength dependent coefficients for $C_{X}=0.32 \mathrm{~g} / \mathrm{L}(\tau=13)$. Taking a flat spectral incident light

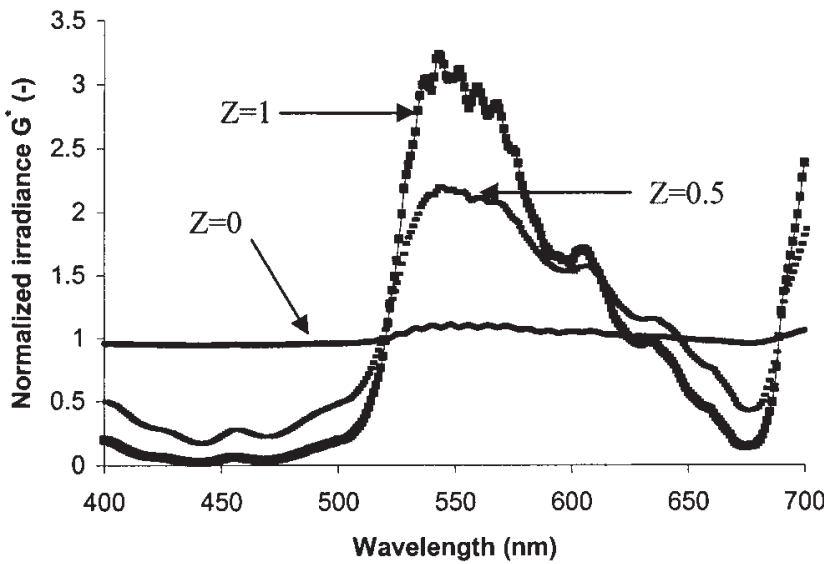

Figure 8. Evolution of the light spectrum quality in the depth of culture $\left(\mathrm{Z}=0, \mathrm{Z}=0.5\right.$ and $\mathrm{Z}=1$ ), for $C_{X}=0.32 \mathrm{~g} / \mathrm{L}$ or $\tau=13$.

flux as already assumed, the spectral irradiance distribution along the z-axis of propagation was calculated from the predictive values of the radiative properties experimentally validated. For convenience, a dimensionless normalized irradiance $G^{*}$ was introduced as follows:

$$
G^{*}(z)=\frac{G_{\lambda_{i}}(z)}{\frac{1}{N} \sum_{N} G_{\lambda_{i}}(z)}=\frac{N G_{\lambda_{i}}(z)}{G_{\Sigma}(z)}
$$

This normalized irradiance is particularly useful to study the spectral quality of light in the photobioreactor because, as seen in Figure 8 for the reduced abscissa $Z=0$, and for a homogeneous distribution in the PAR, the obtained value is one. The slight discrepancies observed around one come from the reflected light at the reactor liquid medium interface which depends on the radiative properties.

When going deeper in the culture, due to wavelength dependent absorption of microalgal suspension, light spectrum is greatly modified from its initial flat distribution; that explains the loss of accuracy when using mean radiative properties averaged on the light spectrum. Note that the determination of the light profile in the depth of culture should be certainly improved by taking into account the real spectrum of fluorescent tubes. But, even in the case of assuming an initial flat spectrum, accurate prediction of light attenuation in the microalgae culture is however obtained. The light spectrum is indeed rapidly modified when going deeper in the culture, and the spectrum of the incident white light has thus only a reduced influence. For a more specific light source (for example, light emitting diodes), consideration of the true emission spectrum should be of more important relevance, as well as the use of spectral coefficients in the predictive model described above. Considering the light conversion processes in the photobioreactor, these results clearly demonstrate that even for quite low optical thicknesses (corresponding to $\mathrm{Z}=0.5$ or $\mathrm{Z}=1$ in Fig. 8), only green light is available in the culture medium with a low capture efficiency, strongly decreasing the local rate of radiant energy absorbed. 
The spectral quality of irradiance (Fig. 8) and the necessity to have an accurate description for the light transfer process in the turbid medium (Fig. 7) are of crucial importance in formulating the local coupling for kinetic rates calculation, and in defining zones of different metabolic activities and stoichiometries from the location of the compensation point in the photobioreactor. This confirms that knowledge predictive models for photobioreactors relies first on a rigorous description of the radiant light transfer which implies that a strong physical basis be used, even for optical and radiative properties calculation, because they can change during cultivation by pigment content modifications, intracellular polymer synthesis, etc.

\section{CONCLUSION}

In this article, a method to rigorously calculate radiant light attenuation profiles in one-dimensional rectangular photobioreactors with a quasi-collimated incidence by a fully predictive approach was investigated and discussed. The basic properties of the cultivated microorganism as shape, size distribution, and pigment content need to be known, and the incident light flux onto the reactor properly determined.

The predictive character and robustness of the proposed approach is ensured by the ability to calculate the spectral optical properties of any microorganism from an in vivo absorption spectrum of pure pigments data bank yet to be developed. This becomes particularly useful when the pigment content of the considered microorganism changes during growth (by combining an increase in light intensity and salinity to trigger massive $\beta$-carotene production with Dunaliella salina, for example), or when an intracellular accumulation of polymers modifies the dielectric behavior of the particle (as stimulation of polyhydroxybutyrate or starch accumulations for hydrogen production), resulting in important modifications in the light transfer process and in the overall photobioreactor running. This powerful method was used and applied to the microalga Chlamydomonas reinhardtii using the Lorenz-Mie theory, which provides the theoretical basis to compute the exact radiative properties of the microorganism, whereas experimental determinations remain very difficult to perform. This overall sequence was then validated by comparison with experimental transmittances obtained with a highly accurate integrating sphere photometer. The good results obtained showed that associating kinetics of intracellular pigment variations with the proposed model could predict the modifications of culture optical properties with time and their influence on the inside light transfer.

Modeling light transfer attenuation in the turbid medium is moreover related to the radiative properties by the radiative transfer equation which must be solved on the photobioreactor with the corresponding boundary conditions. Here, a new solution using the generalized two-flux method while taking into account reflection of light at the rear surface of the reactor was proposed. It was compared with experimental results using mean values on the PAR, averaging either the radiative properties or the spectral irradiances. This latter rigorous approach was proved to better describe the experi- mental measurements, especially at higher optical thicknesses. The simple analytical two-flux approach to describe light transfer in the torus photoreactor cultivating Chlamydomonas reinhardtii up to $\tau=20$, gives very good results. This is probably due to the existence of a quasi-collimated field of radiation and to the spherical shape of this microorganism, giving low values for the back-scattered fraction, but attention must be paid to other microorganisms with different radiative properties. For example, for more complex shapes (cylinders, spheroids, etc.) more sophisticated numerical tools are required to predict radiative properties (Mishchenko et al., 1996) and to calculate light profiles (Kumar et al., 1990; Mengüç and Viskanta, 1983).

Additionally, special attention was paid in the comparison of theoretical calculated variables and experimental determinations and it was demonstrated that using flat cosine or spherical sensors to measure incident light flux densities or spherical irradiances was not a trivial problem. Particularly, the different methods used in this study to characterize the mean incident light flux $q_{0}$ considered as homogeneous on the front side of the reactor are limited to the use of simple twoflux models for radiative transfer. If more efficient methods are envisaged as proposed above, clearly an emission model is needed as proposed by Cassano et al. (1995).

Finally, the fully predictive method proposed seems very efficient as a rigorous tool to obtain an accurate description of the light transfer problem in quasi-collimated irradiated rectangular photobioreactors. It could be used with confidence to formulate the coupling with local kinetics, using metabolic fluxes approach (Cogne et al., 2003), or thermodynamics of irreversible processes (Cornet et al., 1998, 2003) in cases where no important hydrodynamical coupling exists, or using the population balances approach if this latter assumption was not satisfied (Pruvost et al., 2002). The robustness of the resulting knowledge models would then be guaranteed to develop a new design to optimize, or to be used in the model-based predictive and real-time control of photobioreactors.

\section{NOMENCLATURE}

a volumetric absorption coefficient for the turbid medium $\left[\mathrm{m}^{-1}\right]$

$a_{c m} \quad$ volumetric absorption coefficient for the cell material $\left[\mathrm{m}^{-1}\right]$

b backward scattering fraction for the particle [dimensionless]

$C \quad$ cross-section of the particle $\left[\mathrm{m}^{2}\right]$

$C_{C h l-a}$ chlorophyll-a concentration $\left[\mathrm{kg} \mathrm{m}^{-3}\right]$

$C_{C h l-b}$ chlorophyll-b concentration $\left[\mathrm{kg} \mathrm{m}^{-3}\right]$

$C_{P P C}$ photoprotective carotenoid concentration $\left[\mathrm{kg} \mathrm{m}^{-3}\right]$

$C_{\text {pig. } i}$ concentration of pigment $i\left[\mathrm{~kg} \mathrm{~m}^{-3}\right]$

$C_{X} \quad$ biomass concentration $\left[\mathrm{kg} \mathrm{m}^{-3}\right]$

$d_{p} \quad$ mean particle diameter [m]

Ea mass absorption coefficient for the particle $\left[\mathrm{m}^{2} \mathrm{~kg}^{-1}\right]$

$E a_{\text {pig. } i}$ mass absorption coefficient for pigment $i\left[\mathrm{~m}^{2} \mathrm{~kg}^{-1}\right]$

Es mass scattering coefficient for the particle $\left[\mathrm{m}^{2} \mathrm{~kg}^{-1}\right]$

$\mathrm{G} \quad$ spherical irradiance $\left[\mu \mathrm{E} \mathrm{m}^{-2} \mathrm{~s}^{-1}\right]$

I specific radiant intensity $\left[\mu \mathrm{E} \mathrm{m}^{-2} \mathrm{~s}^{-1}\right]$

$1 \quad$ optical path $[\mathrm{m}]$

$L \quad$ photobioreactor depth [m]

$m \quad$ complex refractive index [dimensionless]

$n_{m} \quad$ refractive index of the surrounding medium [dimensionless]

$n \quad$ real part of the refractive index of the particle [dimensionless] 
$N_{P} \quad$ number of particle density $\left[\mathrm{m}^{-3}\right]$

$p \quad$ normalized phase function for scattering of the particle [dimensionless]

PAR photosynthetically active radiation

$Q \quad$ efficiency factor of the particle $\left(Q=C /\left(\pi d_{p}^{2} / 4\right)\right)$ [dimensionless]

$Q^{*} \quad$ packaging function [dimensionless]

$q \quad$ radiant light flux $\left[\mu \mathrm{E} \mathrm{m} \mathrm{m}^{-2} \mathrm{~s}^{-1}\right]$

$r \quad$ sensor radius [m]

$r_{\mathrm{SCN}-}$ volumetric rate of thiocyanate production $\left[\mathrm{mol} \mathrm{m} \mathrm{m}^{-3} \mathrm{~s}^{-1}\right.$ ]

$s \quad$ volumetric scattering coefficient for the turbid medium $\left[\mathrm{m}^{-1}\right]$

$\mathrm{S} \quad$ surface $\left[\mathrm{m}^{2}\right]$

$T \quad$ transmittance [dimensionless]

$V_{32}$ mean efficient volume for the particle from $d_{32}$ sauter diameter $\left[\mathrm{m}^{3}\right]$

$w_{\text {pig. } i}$ mass fraction for pigment $i$ in cell material $\left[\mathrm{kg} \mathrm{kg}^{-1}\right.$ of biomass]

$x \quad$ size parameter for the particle $x=\pi d_{p} / \lambda$ [dimensionless]

$x_{W} \quad$ volume fraction of water in the cell [dimensionless]

$z \quad$ z-abscissa (length) $[\mathrm{m}]$

\section{Greek Letters}

$\alpha \quad$ linear scattering modulus [dimensionless]

$\delta \quad$ two-flux extinction coefficient $\left[\mathrm{m}^{-1}\right]$

$\zeta \quad$ angle [rad]

$\kappa \quad$ imaginary part of the refractive index of the particle [dimensionless]

$\kappa^{*} \quad$ mean imaginary part of the refractive index of the particle in the PAR [dimensionless]

$\lambda \quad$ wavelength [m]

$\theta \quad$ angle between a direction and the abscissa-axis [rad]

$\theta^{\prime} \quad$ angle between a direction and the $\theta$-direction [rad]

$\theta_{c} \quad$ angle between the normal and the incident quasi-collimated beam [rad]

$\xi \quad$ particle absorption optical thickness [dimensionless]

$\xi \quad$ angle (in Appendix) [rad]

$\rho \quad$ reflection coefficient of the stainless steel [dimensionless]

$\rho_{d m} \quad$ density of the dry material in biomass $\left[\mathrm{kg} \mathrm{m}^{-3}\right]$

$\Phi \quad$ quantum yield of conversion [mol $\left.\mathrm{E}^{-1}\right]$

$\phi \quad$ azimuthal angle [rad]

$\tau \quad$ optical thickness [dimensionless]

$\omega \quad$ albedo for single scattering [dimensionless]

$\psi \quad$ photosynthetic photon fluence rate $\left[\mu \mathrm{E} \mathrm{m}^{-2} \mathrm{~s}^{-1}\right]$

\section{Subscripts}

$0 \quad$ relative to incident radiant conditions

$A B S$ relative to the absorption phenomenon

$S C A$ relative to the scattering phenomenon

$\lambda$ relative to a given wavelength

$\Sigma \quad$ denoting spectral values averaged on the light spectrum

\section{Superscripts}

$+\quad$ relative to the positive direction for $\cos (\theta)$

- relative to the negative direction for $\cos (\theta)$

* relative to dimensionless normalized irradiance

\section{APPENDIX}

\section{Exact Calculation for the Photosynthetic Photon Fluence Rate and Comparison With the Photon Spherical Irradiance}

Most of the spherical light sensors actually measure a photosynthetic photon fluence rate $\psi$ (PPFR), as is the case for the LI-COR sensor used in this study (US-SQS Waltz type). Generally, it is supposed that the value given by the sensor is equivalent to the photon spherical irradiance $G$ (PSI), but this is only the case if the radius of the sensor is very small compared to the characteristic size of the reactor. In this Appendix, the true expression of the PPFR from a quasi-collimated two-flux model are established and compared with the PSI corresponding values, and then the main variables affecting this experimental measurement are determined.

It is possible to obtain the rigorous expression of the PPFR for a sensor of radius $r$ from its definition, i.e., the surface integral of the normal hemispherical radiant flux density on the sphere divided by the area of the great circle of this sphere:

$$
\psi=\frac{1}{\pi r^{2}} \oiint \boldsymbol{q}_{\cap} \cdot d \boldsymbol{S}
$$

Considering a linear, one-dimensional anisotropic medium in rectangular coordinates (total thickness $L$ ) and using the quasicollimated two-flux approximation, the integral [Equation (A1)] may be split in two terms corresponding to the positive and negative directions for the intensities (Hottel and Sarofim, 1967). Defining, respectively, the azimuthal angle $\phi$, the angle $\zeta$ between the $z$-axis and a given direction, the angle $\theta_{c}$ for the incident quasi-collimated radiation, then the relative and dimensionless PPFR, normalized by the incident irradiance onto the medium $G_{0}$, is obtained by the following integral:

$$
\begin{aligned}
\frac{\psi}{G_{0}}= & \frac{1}{\pi r^{2}} \int_{0}^{2 \pi}\left[\int_{0}^{\pi / 2} r^{2} \frac{I_{\theta_{c}}^{-}\left(z+r \cos \zeta^{-}\right)}{G_{0}} \cos \xi \sin \xi d \zeta\right. \\
& \left.+\int_{\pi}^{\pi / 2} r^{2} \frac{I_{\theta_{c}}^{+}\left(z+r \cos \zeta^{+}\right)}{G_{0}} \cos \xi \sin \xi d \zeta\right] d \phi
\end{aligned}
$$

In this equation, we defined the new variable $\xi=\zeta-\theta_{c}$ from the quasi-collimated incidence $\theta_{c}$, to keep the plane of symmetry for $\mathrm{I}^{ \pm}\left(\theta_{c}\right)$ perpendicular to the direction $\theta_{c}$ in each integrand. It is then easy to use the two-flux expressions of $\mathrm{I}^{ \pm}$for each hemisphere available from the work of Cornet et al. (1992):

$$
\begin{gathered}
\frac{I^{+}}{I_{0}}=\frac{(1+\alpha)^{2} \exp (-\delta[z-L])-(1-\alpha)^{2} \exp (\delta[z-L])}{(1+\alpha)^{2} \exp (\delta L)-(1-\alpha)^{2} \exp (-\delta L)} \\
\frac{I^{-}}{I_{0}}=\frac{\left(1-\alpha^{2}\right)[\exp (-\delta[z-L])-\exp (\delta[z-L])]}{(1+\alpha)^{2} \exp (\delta L)-(1-\alpha)^{2} \exp (-\delta L)}
\end{gathered}
$$

With $\alpha=\sqrt{\frac{E a}{E a+2 b E s}}, \delta=\sec \left(\theta_{\mathrm{c}}\right) \sqrt{E a(E a+2 b E s)} C_{x}$, and noting that $d \xi=d \zeta$, for an azimuthally independent problem, the double integral [Equation (A2)] becomes:

$$
\begin{aligned}
\frac{\psi}{G_{0}}= & \frac{2\left(S^{-}+S^{+}\right)}{(1+\alpha)^{2} \exp (\delta L)-(1-\alpha)^{2} \exp (-\delta L)} \\
S^{-}= & \int_{0}^{\pi / 2}\left(1-\alpha^{2}\right)\left[\exp \left(-\delta\left[z+r \cos \left(\xi+\theta_{c}\right)-L\right]\right)\right. \\
& \left.-\exp \left(\delta\left[z+r \cos \left(\xi+\theta_{c}\right)-L\right]\right)\right] \cos \xi \sin \xi d \xi \\
S^{+}= & \int_{0}^{\pi / 2}\left[(1+\alpha)^{2} \exp \left(-\delta\left[z+r \cos \left(\xi+\theta_{c}\right)-L\right]\right)\right. \\
& \left.-(1-\alpha)^{2} \exp \left(\delta\left[z+r \cos \left(\xi+\theta_{c}\right)-L\right]\right)\right] \\
& \cos \xi \sin \xi d \xi
\end{aligned}
$$


This rigorous expression for the relative PPFR $\psi / G_{0}$ may be numerically calculated at each location $z$ in the medium, providing the radius of the sensor $r$, the biomass concentration $C_{X}$, and the total thickness of the medium $L$ to be known.

Taking the limit of Equation (A4) when the radius $r$ is assumed very small compared to the thickness $L$ leads to the simple expressions for the $S^{ \pm}$directly:

$$
\begin{aligned}
S^{-}= & \left(1-\alpha^{2}\right)[\exp (-\delta[z-L]) \\
& -\exp (\delta[z-L])] \int_{0}^{\pi / 2} \cos \xi \sin \xi d \xi \\
S^{+}= & {\left[(1+\alpha)^{2} \exp (-\delta[z-L])\right.} \\
& \left.-(1-\alpha)^{2} \exp (\delta[z-L])\right] \int_{0}^{\pi / 2} \cos \xi \sin \xi d \xi
\end{aligned}
$$

in which the integrals are equal to one half, giving with Equation (A3) the expected result for a quasi-collimated field of radiation:

$$
\frac{\psi}{G_{0}}=\frac{I^{-}+I^{+}}{G_{0}}=\frac{G}{G_{0}}
$$

and demonstrating that the sensor, in this case only, gives the exact value of the PSI $G$.

Note that if the incident light flux is normally quasicollimated, then $\theta_{c}=0$, and one has $G / G_{0}=G / q_{0}$; at the opposite, for any other case $G / q_{0}=G / G_{0} \cos \theta_{c}$. Generally speaking, the irradiance profile in a rectangular medium with no reflection at the rear surface is given, for a quasicollimated incidence by (Cornet et al., 1995):

$$
\frac{G}{G_{0}}=2 \frac{(1+\alpha) \exp [-\delta(z-L)]-(1-\alpha) \exp [\delta(z-L)]}{(1+\alpha)^{2} \exp (\delta L)-(1-\alpha)^{2} \exp (-\delta L)}
$$

This equation enables theoretically a comparison between the exact value for the PPFR $\psi$ and the expected one, the PSI $G$, by simply calculating numerically the ratio $\mathrm{G} / \psi$ from Equations (A4) and (A7), thus eliminating $G_{0}$. This work has been done for the considered reactor of this study $(L=0.04 \mathrm{~m})$, for two expected locations of the sensor $(z=0.01$ and $z=0.03 \mathrm{~m}$ ), taking its radius as $1.5 \mathrm{~mm}$ corresponding to the Waltz-LI COR sensor used and described in the Materials and Methods section. The obtained results, taking a normal incidence for the incident light flux, are given in Figure A1 where the ratio G/ $\psi$ has been plotted vs. the biomass concentration. Interestingly, the results show that the ratio decreases when the biomass concentration increases, and is only 1 for the limiting case of dilute solution, whereas the location of the sensor appears to have no significant effect. The observed discrepancies increasing the biomass concentration probably come from the existence of a marked light gradient onto the spherical sensor. Special attention must then be paid to this problem because, even for a very low-sized sensor ( $3 \mathrm{~mm}$ diameter), the true PSI may be

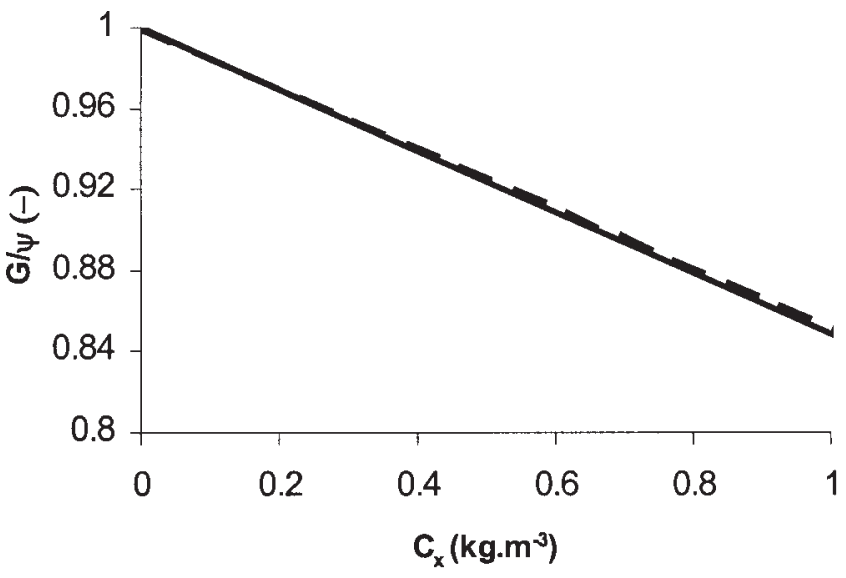

Figure A1. Ratio of the photosynthetic photon fluence rate to the photon spherical irradiance with respect to the biomass concentration ( $z=0.01 \mathrm{~cm},-z=0.03 \mathrm{~cm}$ ).

overestimated by $15 \%$ at a biomass concentration of $1 \mathrm{~g} / \mathrm{L}$. Nevertheless, considering maximal values of $0.5 \mathrm{~g} / \mathrm{L}$ reported here, the approximation made by all the manufacturers taking $G=\psi$ can be considered as relevant with an accuracy higher than $7 \%$ which is in agreement with nominal sensor accuracy and is the accuracy generally obtained for any biological determination at the reactor scale.

\section{References}

Acien Fernandez FG, Garcia Camacho F, Sanchez Perez JA, Fernandez Sevilla JM, Molina Grima E. 1997. A model for light distribution and averaged solar irradiance inside outdoor tubular photobioreactors for the microalgal mass culture. Biotechnol Bioeng 55:701-714.

Aiba S. 1982. Growth kinetics of photosynthetic microorganisms. Adv Biochem Eng 23:85-156.

Bidigare R, Ondrusek M, Morrow J, Kiefer D. 1990. In vivo absorption properties of algal pigments. Proceedings of SPIE, Ocean optics X 1302:290-302.

Bohren CF, Huffman DR. 1983. Absorption and Scattering of light by small particles. New York: Wiley.

Cassano AE, Martin CA, Brandi RJ, Alfano OM. 1995. Photoreactor analysis and design: fundamentals and applications. Ind Eng Chem Res 34: $2155-2201$.

Chandrasekhar S. 1960. Radiative transfer. New York: Dover.

Cogne G, Gros J-B, Dussap CG. 2003. Identification of a metabolic network structure representative of arthrospira platensis metabolism. Biotechnol Bioeng 84:667-676.

Cornet J-F, Albiol J. 2000. Modeling photoheterotrophic growth kinetics of Rhodospirillum rubrum in rectangular photobioreactors. Biotechnol Prog 16:199-207.

Cornet J-F, Dussap C-G, Dubertret G. 1992. A structured model for simulation of cultures of the cyanobacterium Spirulina platensis in photobioreactors: I. Coupling between light transfer and growth kinetics. Biotechnol Bioeng 40:817-825.

Cornet J-F, Dussap C-G, Gros J-B. 1994. Conversion of radiant light energy in photobioreactors. AIChE J 40(6): 1055-1066.

Cornet J-F, Dussap C-G, Gros J-B. 1995. A simplified monodimensional approach for modeling coupling between radiant light transfer and growth kinetics in photobioreactors. Chem Eng Science 50(9):1489-1500.

Cornet J-F, Dussap C-G, Gros J-B. 1998. Kinetics and energetics of photosynthetic micro-organisms in photobioreactors: Application to Spirulina growth. Adv Biochem Eng/Biotechnol 59:155-224. 
Cornet J-F, Dussap C-G, Leclercq J-J. 2001. Simulation, design and model based predictive control of photobioreactors. In: Hofman M and Thonart P, eds. Focus on Biotechnology 4: Engineering and Manufacturing for Biotechnology. Dordrecht: Kluwer Academic p 227-238.

Cornet J-F, Favier-Teodorescu L, Dussap C-G. 2003. Modelling stability of photoheterotrophic continuous cultures in photobioreactors. Biotechnol Prog 19:1216-1227.

Cornet J-F, Marty A, Gros J-B. 1997. Revised technique for the determination of mean incident light fluxes on photobioreactors. Biotechnol Prog 13:408-415.

Csogör Z, Herrenbauer M, Schmidt K, Posten C. 2001. Light distribution in a novel photobioreactor-Modelling for optimization. J Appl Phycol 13: $325-333$.

Harris E. 1989. The Chlamydomonas sourcebook: A comprehensive guide to biology and laboratory use. San Diego: Academic Press, Inc.

Hottel HC, Sarofim AF. 1967. Radiative transfer in an absorbing-scattering medium. In Radiative transfer. New York: McGraw-Hill. p 408-437.

Janssen M, Janssen M, de Winter M, Tramper J, Mur LR, Snel J, Wijfels RH. 2000. Efficiency of light utilization of Chlamydomonas reinhardtii under medium-duration light/dark cycles. J Biotechnol 78:123-137.

Kumar S, Majumdar A, Tien CL. 1990. The differential discrete ordinate method for solutions of the equation of radiative transfer. Journal of Heat Transfer 112:424-429.

Leifer A. 1988. The kinetics of environmental aquatic photochemistry, theory and practice. Washington, DC: American Chemical Society.

Melis A, Liping Z, Forestier M, Ghirardi ML, Seibert M. 2000. Sustained photobiological hydrogen gas production upon reversible inactivation of oxygen evolution in the green alga Chlamydomonas reinhardtii. Plant Physiol 122:127-135.

Mengüç MP, Viskanta R. 1983. Comparison of radiative transfer approximations for a highly forward scattering planar medium. J Quant Spectrosc Radiat Transfer 29:381-394.

Mishchenko MI, Travis LD, Mackowski DW. 1996. T-matrix computations of light scattering by nonspherical particles: a review. J Quant Spectrosc Radiat Transfer 55:535-575.
Morel A, Bricaud A. 1981. Theoretical results concerning light absorption in a discrete medium, and application to specific absorption of phytoplankton. Deep Sea Research 28A:1375-1393.

Pruvost J, Legrand J, Legentilhomme P, Muller-Feuga A. 2002. Simulation of microalgae growth in limiting light conditions-flow effect. AIChE J 48:1109-1120.

Pruvost J, Legrand J, Legentilhomme P, Rosant J-M. 2004. Numerical investigation of bend and torus flows. Part II: Flow simulation in torus reactor. Chem Eng Sci 59:3359-3370.

Sanchez Miron A, Ceron Garcia MC, Contreras Gomez A, Garcia Camacho F, Molina Grima E, Chisti Y. 2003. Shear stress tolerance and biochemical characterization of Phaeodactylum tricornutum in quasi steady-state continuous culture in outdoors photobioreactors. Biochem Eng J 16(3):287-297.

Siegel R, Howell J-R. 2002. Thermal radiation heat transfer, 4th ed. New York: Taylor and Francis.

Spadoni G, Bandini E, Santarelli F. 1978. Scattering effects in photosensitized reactions. Chem Eng Sci 33:517-524.

Stramigioli C, Spiga G, Santarelli F. 1982. Radiative transfer and batch photochemical reaction in a plan slab. Ind Eng Chem Fundam 21:119123.

Strickland JDH, Parsons TR. 1968. A practical handbook of seawater analysis: Pigment analysis. Bulletin of Fisheries Research Board of Canada 167. Ottawa: Queen's Printer.

Suh IS, Lee SB. 2003. A light distribution model for an internally radiating photobioreactor. Biotechnol Bioeng 82:180-189.

Tredici MR, Zitelli GC. 1998. Efficiency of sunlight utilization: Tubular versus flat photobioreactors. Biotechnol Bioeng 57:187-197.

Van de Hulst HC. 1981. Light scattering by small particles, 2nd ed. Mineola, NY: Dover.

Wyatt PJ. 1968. Differential light scattering: a physical method for identifying living bacterial cells. Applied Optics 7:1879-1896.

Yun Y-S, Park JM. 2003. Kinetic modeling of the light-dependent photosynthetic activity of the green microalga Chlorella vulgaris. Biotechnol Bioeng 83:303-311. 